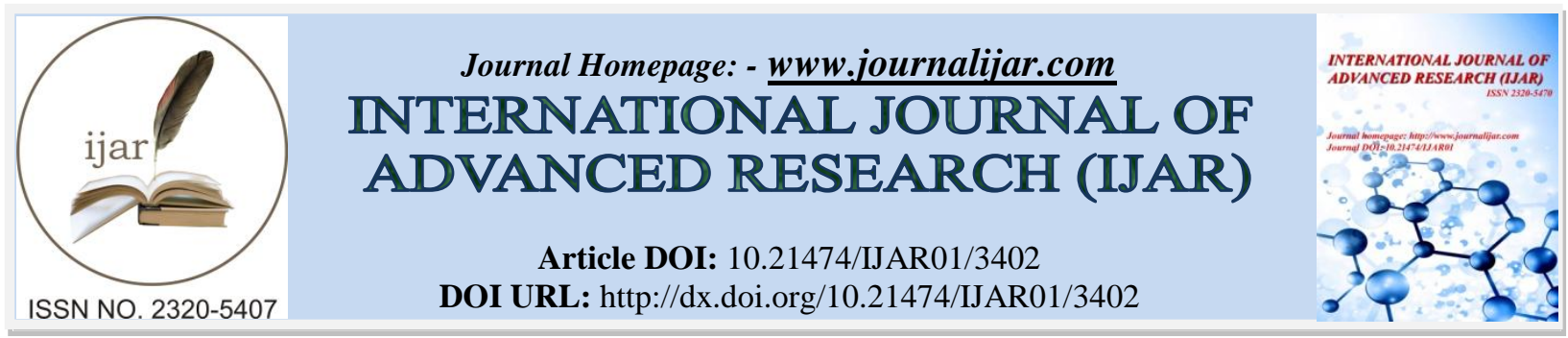

RESEARCH ARTICLE

\title{
ANALYSIS OF ACHIEVEMENT MOTIVATION AND AGGRESSION AMONG THE ATTACKER, SETTER AND LIBERO PLAYER AT INTER COLLEGIATE LEVEL MEN VOLLEYBALL PLAYERS.
}

Basanagouda Laxmeshwar.

Assistant Professor Physical Education, College of Rural Home Science, University of Agricultural Sciences, Dharwad.

\section{Manuscript Info}

\section{Manuscript History}

Received: 18 December 2016

Final Accepted: 22 January 2017

Published: February 2017

Key words:-

Achievement Motivation, Aggression, Attacker, Setter, Libero and Volleyball.

\section{Abstract}

"The life is like sport. The lord is the greatest sportsman. The purpose of the study was to analysis of achievement motivation and aggression among attacker, setter, and libero player at inter collegiate level volleyball players. It was hypothesized that there will be a significant of achievement motivation and aggression among the attacker, setter, and libro player at inter collegiate level volleyball players. A total of 90 inter collegiate level consist of attacker setter and libero are selected. Selected as a random, they were divided in to 3 equal groups ( 30 from attacker, 30 from setter and 30 from libero) The research scholar reviewed the available scientific literature, books, journals, periodicals, and magazine and research papers pertaining to the study. Taking into consideration of the importance of these variables and the feasibility criteria for these following variables were selected for the investigator. 1. Achievement motivation 2. Aggression The data that were collected form the subjects were treated statistically. To find out the significance difference among the attacker, setter and libero with their achievement motivation and aggression for that Analysis of Variance (ANOVA) was used to find out any significant difference among the group. In view of the formulation of hypotheses the following results were emphasized based on the analysis of data The result of the study shows that there has no significant difference in the achievement motivation among attacker, setter, libero between these variables have better in achievement motivation. The result of the study showed that there has significant difference in the aggression among attacker, setter \& libero at inter collegiate level men volley ball players Because volley players need very quick movement for receiving and attacker the ball every second they keep on moving place to place quickness is a prime necessity in the modem volley bale both in attack and defence. That is the reason attacker, setter and libero player hove more aggressive in game. 


\section{Introduction:-}

Sport has become one of the most widely practiced human activities Whether as professionals of amateurs, regularly or occasionally, millions of people now engage in the various forms of physical and sporting activity available in the European Union. In addition to its health benefits, sport plays an important economic and social role. It contributes to social integration and inclusion, plays a part in the process of non-formal education, promotes intercultural exchanges and creates. "The life is like sport. The lord is the greatest sportsman. He plays without thinking of winning or losing". Physical Education is an integral part of total Education. It is an education through physical Fitness, Social Fitness, moral fitness and emotional fitness for an individual to develop not only a good physique but also help in develop desirable social qualities. The word 'Sports' occasionally denotes either to a pleasant part time or somewhat hazardous recreation. Today sports are considered as international discipline as it develops international understanding and universal brotherhood. Sports develop national character and also it provides to fullest self expression to man and it is one of the fundamental needs. RAMA DASS (2003):Conducted a study on a comparative study on self concept and achievement motivation between physical education and education student students. Mukta Rani Rastogi's self concept scale was used to quanti self concept and M.L.kamelsh sports achievement motivation test questionnaire was used. The purpose of the study was analysis the self concept and achievement motivaton between physical education and education students. To achieve this purpose a totally 30 subjects from physical education and education. Their age group is between 20 to 25 years, the 't'-ratio was used the results of the study was the level of the self-concept between physical education students was higher than education students, the level of achievements motivation between physical education students was higher than education student.

\section{Sports:-}

The word 'Sports' occasionally denotes either to a pleasant part time or somewhat hazardous recreation.

\section{Sport Psychology:-}

Sports psychologist is the scientific study of people and their behaviours in sport. The main job of a sports psychologist is to recognize how participation in sport exercise and physical activity enhances a person's development. Sport psychology is a specialization within psychology that seeks to understand psychological/mental factors that affect performance in sports, physical activity and exercise and apply these to enhance individual and team performance.

\section{Motivation:-}

Ask any person who is successful in whatever he or she is doing What motivate him/her, and very likely answer will be "goals". Goal Setting goals, is extremely important to motivation and success.

\section{Aggression:-}

Aggression refers to any behaviour that is hostiles, destructive and/or violent. Generally, aggressive behaviour has the potential to Inflict injury or damage to the target person or object. Example of aggressive behaviour include physical assault, throwing objects, property destruction, self harming behaviours, and verbal threats.

\section{Volleey Ball:-}

Volleyball is a typical American game. It was invented by "William,G,Moran" Physical Director of Holyoake YMCA Mass, U.S.A. in the year 1895.he wanted to introduce a game to the members of his YMCA with a view to provide a suitable recreational game less strenuous then that of Basket Ball. There was at the time, a game called "Minton" in which at worsted yarn ball was batted back and forth over a 7 foot net with help of a racket. Morgan modified this game by eliminating the rocket and worsted yarn ball and experimented with an inflected basketball bladder, which was batted with the hands on over the net. He introduced this game in his gymnasium and he called this game as 'Mintonette'. Since the basketball bladder was not conducive for proper play, a new ball devised though the help of a sports company. Dr.A.T.Haisteds of spring Field College, gave the application of "Volley Ball" to this game, since the idea of the play was to volley the ball to and fro over the net.

\section{Statement Of The Problem:-}

The purpose of the study was to analysis of achievement motivation and aggression among attacker, setter, and libero player at inter collegiate level volleyball players. 


\section{Hypothesis:-}

It was hypothesized that there will be a significant of achievement motivation and aggression among the attacker, setter, and libro player at inter collegiate level volleyball players.

\section{Delimitation:-}

The present study was delimited in the following aspects.

1. The study will be restricted to 30 attacker, 30 setter, 30 libero players.

2. The age limit of the subject will be limited to the range of 18 to 25 years.

3. The study was restricted to two psychological variables namely achievement motivation and aggression were analyzed.

4. Only standardized questionnaire was measured the psychological variables.

a. Anandakumar inventor for aggression

b. M.L.Kamalesh questionnaire for achievement motivation.

\section{Limitation:-}

The limitation of the present study is as follows.

1. The food habits, other regular habits and life style are not controlled.

2. The regular activities of the students will not be controlled.

3. Family background of the subject will not be considered.

4. Environmental factors, which contribute to the mental ability of the players, were not talent into consideration.

5. The response of the subject to the questionnaire might not be honest in all cases and this was recognized as a limitation.

\section{Significance of The Study:-}

1. The study will be helping the players to find out psychological factors.

2. The study will help the coaches.

\section{Definition of Terms:-}

Aggression:-

"Defines aggression as the intentional response a person makes to inflict pain or harm on another person"

\section{Achievement Motivation:-}

Achievement motivation is an effective arousal state directing behaviour in an achievement oriented activity cognitively appraised as potentially satisfying.

\section{Attacker:-}

An offensive shot where a player returns the ball to the opponent's court. Normally this is done in a manner where the attacker hits the ball above the height of the net in a downward direction toward the floor.

\section{Setter:-}

Setters have the task for orchestrating the offense of the team. They aim for second touch and their main responsibility is to place the ball in the air where the attacker can hit the ball into the opponents' court in the easiest way possible. They have to be able to operate with the hitters with variety and break up the enemy's block. Setters need to have swift and skilful appraisal and tactical accuracy, and must be quick at moving around the court.

\section{Libero:-}

"Lee beh rob" - Lee — means to act against the wind from same side of the two part and dictionary word.

\section{Methodology:-}

Selection Of Subjects:-

A total of 90 inter collegiate level consist of attacker setter and libero are selected. Selected as a random, they were divided in to 3 equal groups ( 30 from attacker, 30 from setter and 30 from libero) 


\section{Selection of Variables:-}

The research scholar reviewed the available scientific literature, books, journals, periodicals, and magazine and research papers pertaining to the study. Taking into consideration of the importance of these variables and the feasibility criteria for these following variables were selected for the investigator.

1. Achievement motivation

2. Aggression

Psychological Variables:-

Achievement motivation laws measured by using questionnaire.

\section{Achievement Motivation:-}

Motivation was measured through achievement motivation questionnaire prepared by M. L.Kamlesh. Achievement motivation was given to all investigation; the computed questionnaire was scored as follows.

For items 1,3,4,9,10,11,12,13,16,17 and 20 the despondence answer "a" scored two points: if he answer "B" scored only zero points.

For items $2,5,6,7,8,14,15,18$, and 19 the despondence answer "b" is scores 2 points, if he answer "a" scored only zero points.

The larger the score, higher the achievement motivation of the subject

\section{Aggression:-}

To measure the aggression Anandakumar aggression scale prepared by crafty was used.

Aggression was given to all investigation, The computed questionnaire was scored as follows.

For items $1,4,5,6,9,12,14,16,18,21,22,24$, and 25 answer "yes" he scored two point. In the answer "no" get zero point.

For items 2,3,7,8,10,11,13,15,17,19,20, and 23 answer "no" he scored two point. If he answer "yes" zero point.

( The copy of questionnaire was given in appendix-II )

\section{Statistical Techniques:-}

The data that were collected from the subjects were treated statistically. To find out the significance difference among the attacker, setter and libero with their achievement motivation and aggression for that Analysis of Variance (ANOVA) was used to find out any significant difference among the group.

\section{Analysis and Interpretation Of Data:-}

After the data had been collected, it was processed and tabulated using Microsoft Excel - 2000 Software. The data collected on achievement motivation and aggression from three types of players (Attackers, Setter and Libero) at inter collegiate men Volleyball players. Then the data were analyzed with reference to the objectives and hypotheses by using differential statistics including One way ANOVA followed by unpaired t-test and Pearson's correlation coefficient technique by using SPSS 16.0 statistical software and the results obtained thereby have been interpreted.

Hypothesis: There is no significant difference between three groups of players (Attackers, Setter and Libero) at inter collegiate men Volleyball players respect to achievement motivation scores.

To achieve this hypothesis, the one way NAOVA test was applied and the results are presented in the following table.

Table: Results of ANOVA test between three groups of players (Attackers, Setter and Libero) at inter collegiate men Volleyball players respect to achievement motivation scores

\begin{tabular}{|l|l|l|l|l|l|}
\hline Source of variation & Degrees of freedom & Sum of squares & Mean sum of squares & F-value & P-value \\
\hline Between groups & 2 & 86.67 & 43.33 & 3.4596 & $0.0358^{*}$ \\
\hline Within groups & 87 & 1089.73 & 12.53 & & \\
\hline Total & 89 & 1176.40 & & & \\
\hline
\end{tabular}

$* \mathrm{p}<0.05$ From the results of the above table it can be seen that, the three groups of players (Attackers, Setter and Libero) at inter collegiate men Volleyball players differ statistically significant respect to achievement motivation 
scores $(\mathrm{F}=3.4596, \mathrm{p}<0.05)$ at $5 \%$ level of significance. Hence, the null hypothesis is rejected and alternative hypothesis is accepted. It means that, the attacker players have significant higher achievement motivation scores as compared to Setter and Libero players at inter collegiate men Volleyball players. The mean of achievement motivation scores are also presented in the following figure.

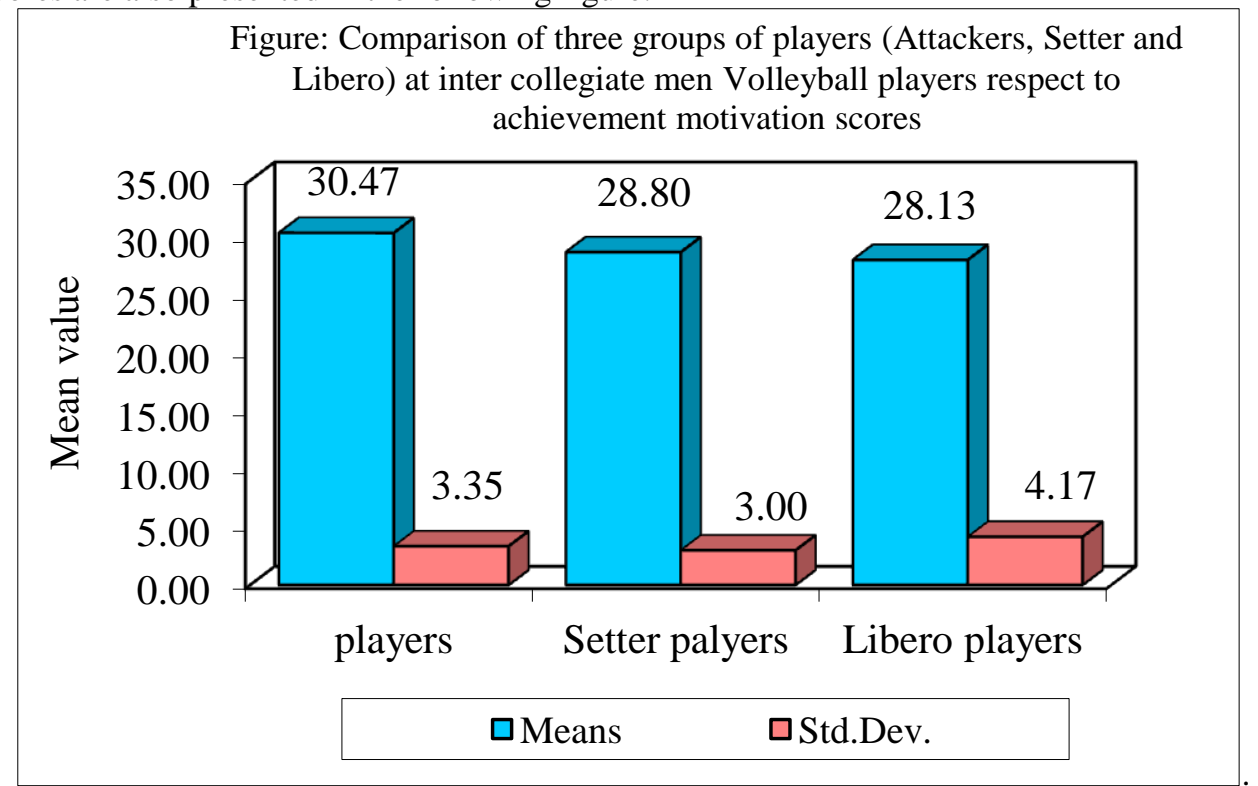

Sub Hypothesis: There is no significant difference between Attackers and Setter players at inter collegiate men Volleyball players respect to achievement motivation scores.

To achieve this hypothesis, the t- test was applied and the results are presented in the following table.

Table: Results of t-test between Attackers and Setter players at inter collegiate men Volleyball players respect to achievement motivation scores

\begin{tabular}{|l|l|l|l|l|}
\hline Players & Mean & SD & t-value & p-value \\
\hline Attacker player & 30.4667 & 3.3501 & 2.0303 & $0.0469^{*}$ \\
\hline Setter player & 28.8000 & 2.9989 & & \\
\hline
\end{tabular}

$* \mathrm{p}<0.05$ From the results of the above table it can be seen that, the Attackers and Setter players at inter collegiate men Volleyball players differ statistically significant respect to achievement motivation scores $(\mathrm{t}=2.0303, \mathrm{p}<0.05)$ at $5 \%$ level of significance. Hence, the null hypothesis is rejected and alternative hypothesis is accepted. It means that, the attacker players have significant higher achievement motivation scores as compared to Setter players at inter collegiate men Volleyball players. The mean of achievement motivation scores are also presented in the following figure.

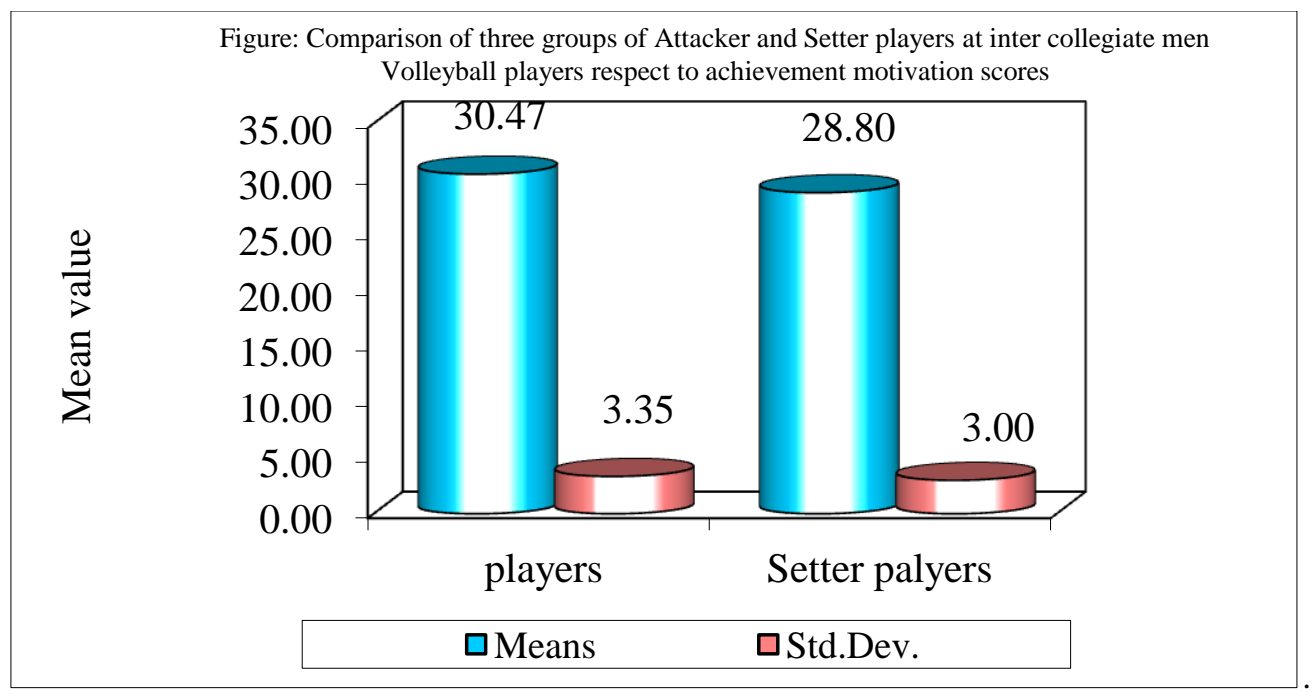


Sub Hypothesis: There is no significant differences between Attacker and Libero players at inter collegiate men Volleyball players respect to achievement motivation scores.

To achieve this hypothesis, the t- test was applied and the results are presented in the following table.

Table: Results of t-test between Attacker and Libero players at inter collegiate men Volleyball players respect to achievement motivation scores

\begin{tabular}{|l|l|l|l|l|}
\hline Players & Mean & SD & t-value & p-value \\
\hline Attacker player & 30.4667 & 3.3501 & 2.3904 & $0.0201 *$ \\
\hline Libero player & 28.1333 & 4.1666 & & \\
\hline
\end{tabular}

$* \mathrm{p}<0.05$ From the results of the above table it can be seen that, the Attackers and Lebero players at inter collegiate men Volleyball players differ statistically significant respect to achievement motivation scores $(t=2.3904, p<0.05)$ at $5 \%$ level of significance. Hence, the null hypothesis is rejected and alternative hypothesis is accepted. It means that, the attacker players have significant higher achievement motivation scores as compared to Lebero players at inter collegiate men Volleyball players. The mean of achievement motivation scores are also presented in the following figure.

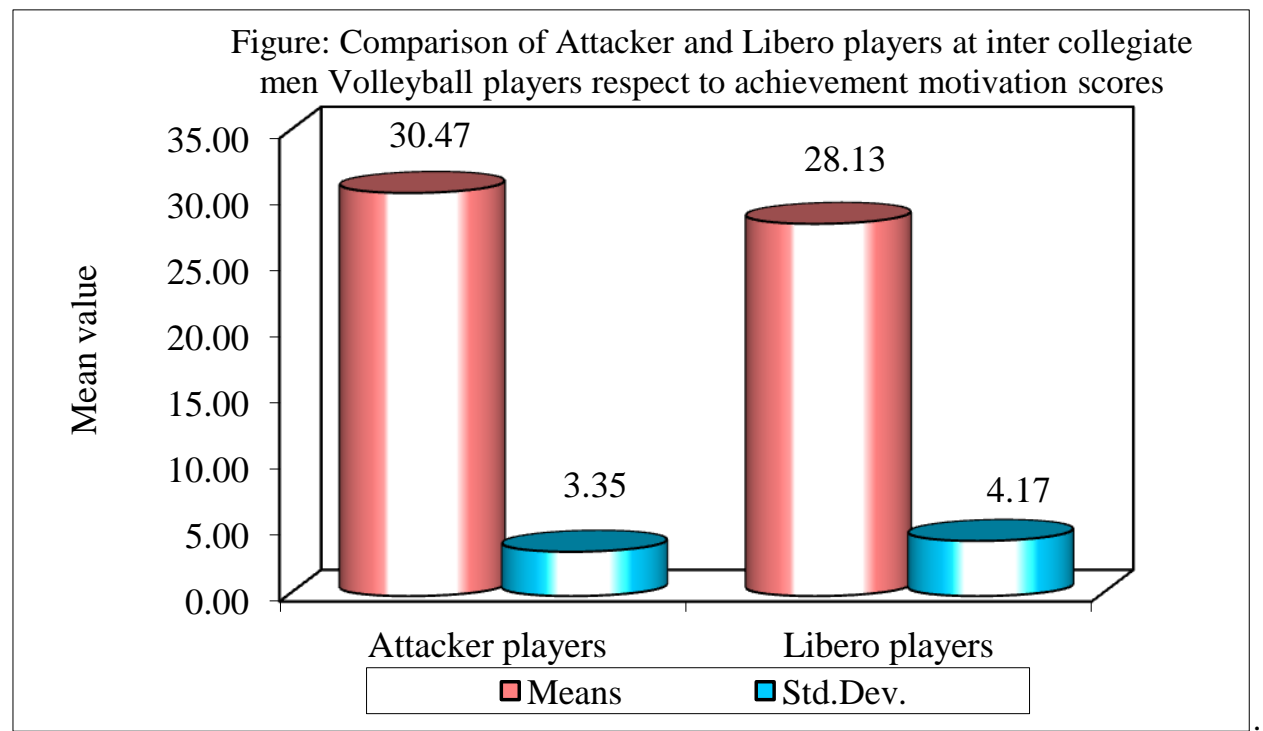

Sub Hypothesis: There is no significant differences between Setter and Libero players at inter collegiate men Volleyball players respect to achievement motivation scores.

To achieve this hypothesis, the t- test was applied and the results are presented in the following table.

Table: Results of t-test between Setter and Libero players at inter collegiate men Volleyball players respect to achievement motivation scores

\begin{tabular}{|l|l|l|l|l|}
\hline Players & Mean & SD & t-value & p-value \\
\hline Setter player & 28.8000 & 2.9989 & 0.7113 & 0.4798 \\
\hline Libero player & 28.1333 & 4.1666 & & \\
\hline
\end{tabular}

From the results of the above table it can be seen that, the Atta Setter and Libero players at inter collegiate men Volleyball players do not differ statistically significant respect to achievement motivation scores $(t=0.7113, p>0.05)$ at $5 \%$ level of significance. Hence, the null hypothesis is accepted and alternative hypothesis is rejected. It means that, the Setter and Libero players at inter collegiate men Volleyball players respect to achievement motivation scores have similar achievement motivation scores. The mean of achievement motivation scores are also presented in the following figure. 


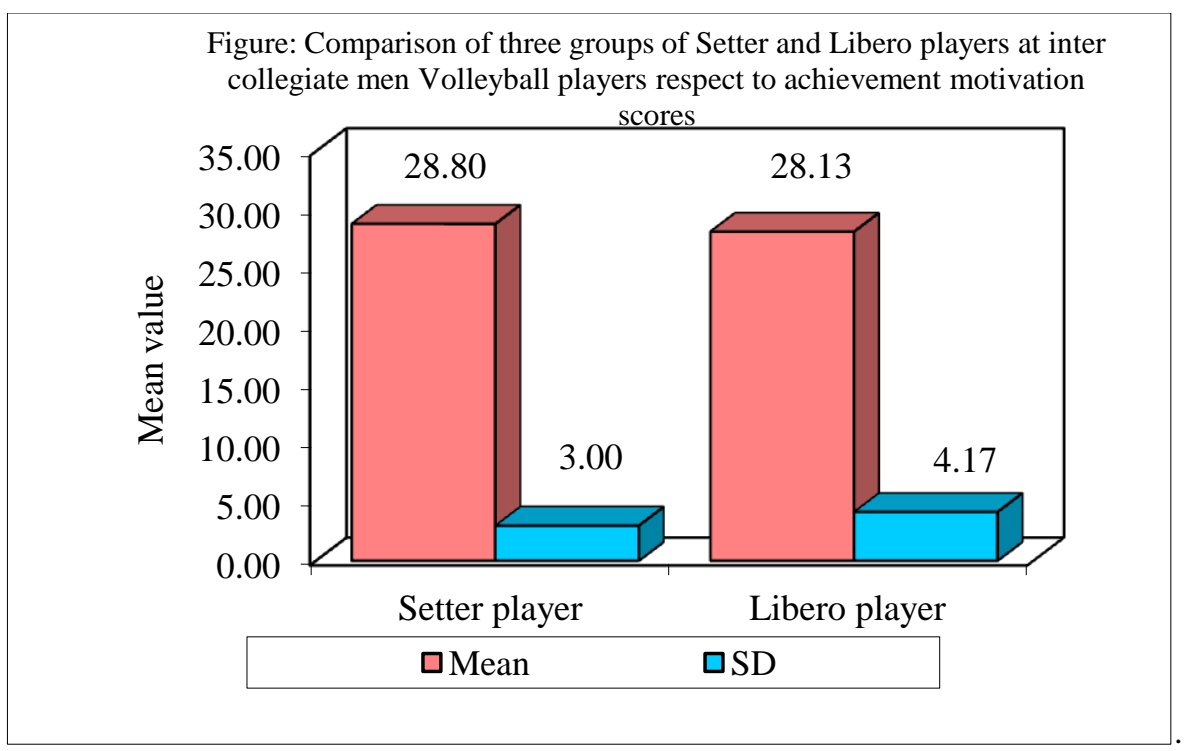

Hypothesis: There is no significant difference between three groups of players (Attacker, Setter and Libero) at inter collegiate men Volleyball players respect to aggression scores.

To achieve this hypothesis, the one way NAOVA test was applied and the results are presented in the following table.

Table: Results of ANOVA test between three groups of players (Attacker, Setter and Libero) at inter collegiate men Volleyball players respect to aggression scores

\begin{tabular}{|l|l|l|l|l|l|}
\hline Source of variation & Degrees of freedom & Sum of squares & Mean sum of squares & F-value & P-value \\
\hline Between groups & 2 & 297.60 & 148.80 & 9.6898 & $0.0002^{*}$ \\
\hline Within groups & 87 & 1336.00 & 15.36 & & \\
\hline Total & 89 & 1633.60 & & & \\
\hline
\end{tabular}

$* \mathrm{p}<0.05$ From the results of the above table it can be seen that, the three groups of players (Attackers, Setter and Libero) at inter collegiate men Volleyball players differ statistically significant respect to aggression scores $(\mathrm{F}=9.6898, \mathrm{p}<0.05)$ at $5 \%$ level of significance. Hence, the null hypothesis is rejected and alternative hypothesis is accepted. It means that, the attacker players have significant higher aggression scores as compared to Setter and Libero players at inter collegiate men Volleyball players. The mean of aggression scores are also presented in the following figure.

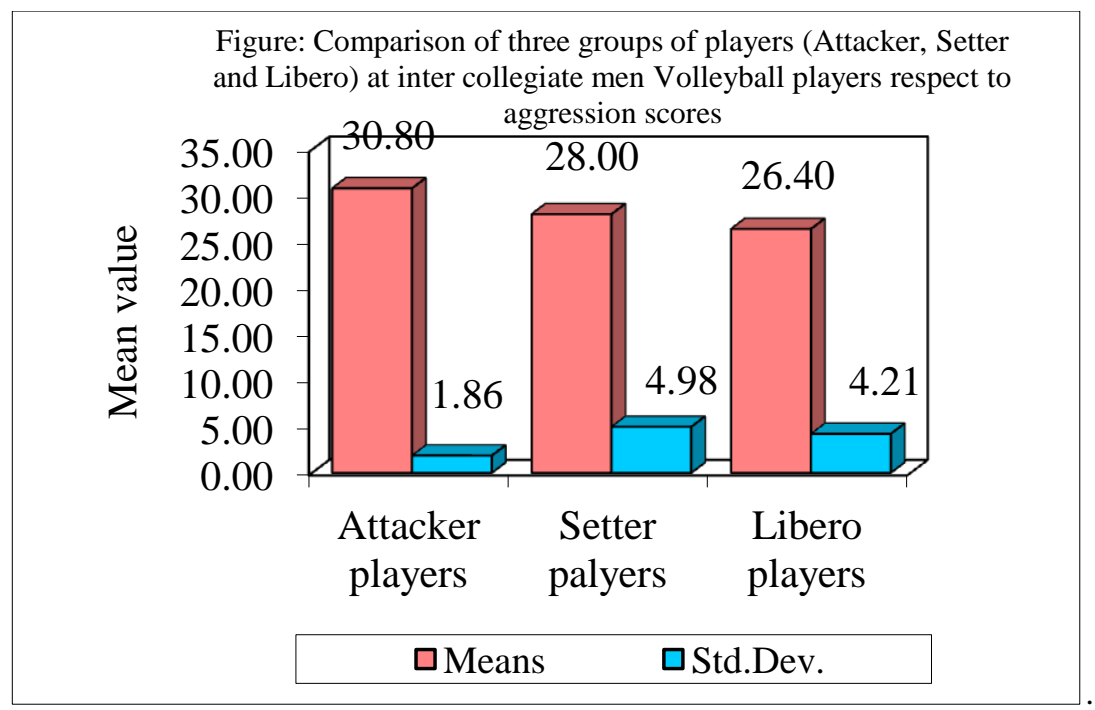

Sub Hypothesis: There is no significant differences between Attacker and Setter players at inter collegiate men Volleyball players respect to aggression scores. 
To achieve this hypothesis, the t- test was applied and the results are presented in the following table.

Table: Results of t-test between Attacker and Setter players at inter collegiate men Volleyball players respect to aggression scores

\begin{tabular}{|l|l|l|l|l|}
\hline Players & Mean & SD & t-value & p-value \\
\hline Attacker player & 30.8000 & 1.8644 & 2.8827 & $0.0055^{*}$ \\
\hline Setter player & 28.0000 & 4.9827 & & \\
\hline
\end{tabular}

*p $<0.05$ From the results of the above table it can be seen that, the Attackers and Setter players at inter collegiate men Volleyball players differ statistically significant respect to aggression scores $(t=2.8827, p<0.05)$ at $5 \%$ level of significance. Hence, the null hypothesis is rejected and alternative hypothesis is accepted. It means that, the attacker players have significant higher aggression scores as compared to Setter players at inter collegiate men Volleyball players. The mean of aggression scores are also presented in the following figure.

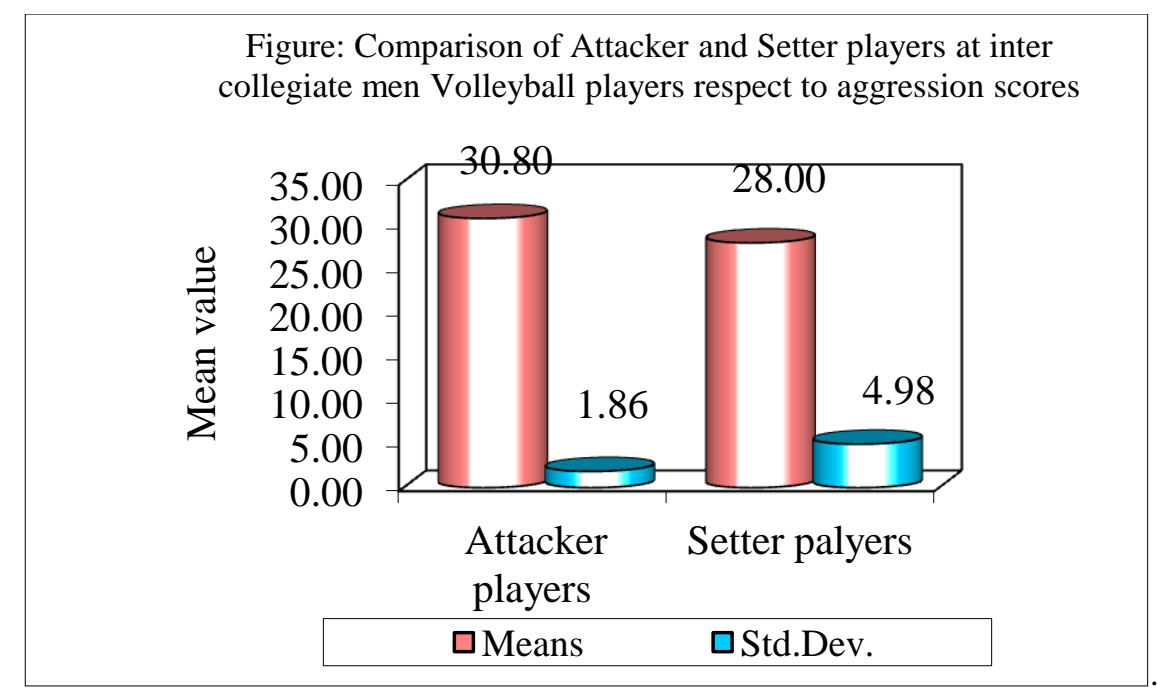

Sub Hypothesis: There is no significant difference between Attacker and Libero players at inter collegiate men Volleyball players respect to aggression scores.

To achieve this hypothesis, the t- test was applied and the results are presented in the following table.

Table: Results of t-test between Attacker and Libero players at inter collegiate men Volleyball players respect to aggression scores

\begin{tabular}{|l|l|l|l|l|}
\hline Players & Mean & SD & t-value & p-value \\
\hline Attacker player & 30.8000 & 1.8644 & 5.2290 & $0.0000^{*}$ \\
\hline Libero player & 26.4000 & 4.2149 & & \\
\hline
\end{tabular}

* $\mathrm{p}<0.05$ From the results of the above table it can be seen that, the Attackers and Lebero players at inter collegiate men Volleyball players differ statistically significant respect to aggression scores $(t=5.2290, \mathrm{p}<0.05)$ at $5 \%$ level of significance. Hence, the null hypothesis is rejected and alternative hypothesis is accepted. It means that, the attacker players have significant higher aggression scores as compared to Lebero players at inter collegiate men Volleyball players. The mean of aggression scores are also presented in the following figure. 


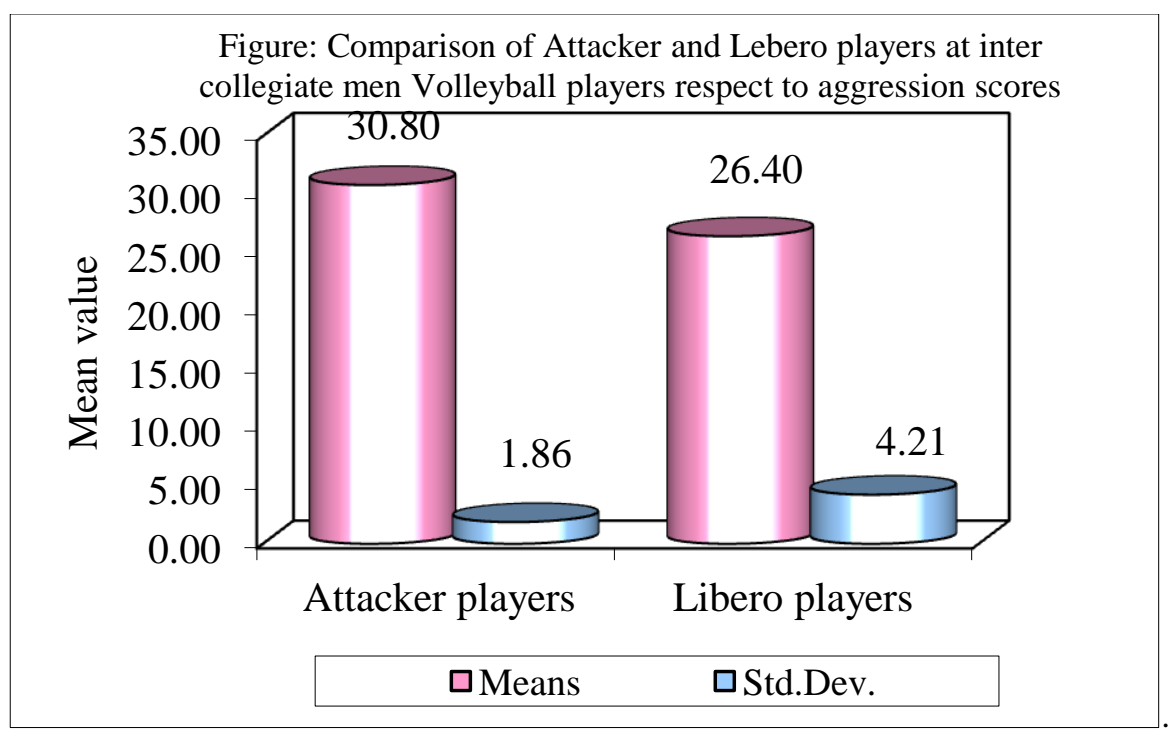

Sub Hypothesis: There is no significant differences between Setter and Libero players at inter collegiate men Volleyball players respect to aggression scores.

To achieve this hypothesis, the t- test was applied and the results are presented in the following table.

Table: Results of t-test between Setter and Libero players at inter collegiate men Volleyball players respect to aggression scores

\begin{tabular}{|l|l|l|l|l|}
\hline Players & Mean & SD & t-value & p-value \\
\hline Setter player & 28.0000 & 4.9827 & 1.3428 & 0.1846 \\
\hline Libero player & 26.4000 & 4.2149 & & \\
\hline
\end{tabular}

From the results of the above table it can be seen that, the Atta Setter and Libero players at inter collegiate men Volleyball players do not differ statistically significant respect to aggression scores $(t=1.3428, p>0.05)$ at $5 \%$ level of significance. Hence, the null hypothesis is accepted and alternative hypothesis is rejected. It means that, the Setter and Libero players at inter collegiate men Volleyball players respect to aggression scores have similar aggression scores. The mean of aggression scores are also presented in the following figure.

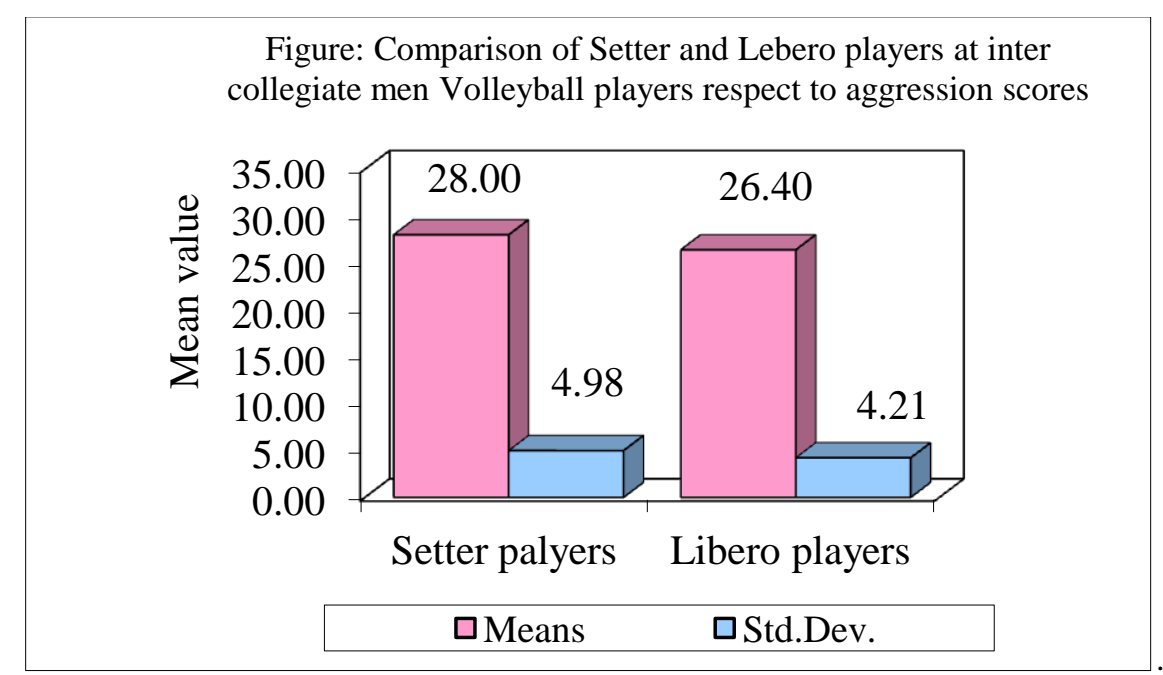

Hypothesis: There is no significant association between achievement motivation and aggression scores of all players i.e. Attacker, Setter and Libero at inter collegiate men Volleyball players 
To achieve this hypothesis, the Karl Pearson's correlation coefficient technique has been applied and the results are presented in the following table.

Table: Results of correlation coefficient between achievement motivation and aggression scores of all players i.e. Attacker, Setter and Libero at inter collegiate men Volleyball players.

\begin{tabular}{|l|l|l|l|}
\hline \multirow{2}{*}{ variables } & \multicolumn{3}{|l|}{ Correlation coefficient between achievement motivation with } \\
\cline { 2 - 4 } & Correlation coefficient & t-value & P-value \\
\hline Aggression scores & 0.2880 & 2.8208 & $0.0059^{*}$ \\
\hline
\end{tabular}

$* \mathrm{p}<0.05$ From the results of the above table, it can observed that, a significant and positive relationship was observed between achievement motivation and aggression scores of all players i.e. Attacker, Setter and Libero at inter collegiate men Volleyball players $(\mathrm{r}=0.2880, \mathrm{p}<0.05)$ at $5 \%$ level of significance. Hence, the null hypothesis is rejected and alternative hypothesis is accepted. It means that, the achievement motivation scores are increases or decreases with increase or decrease in aggression scores of all players.

Hypothesis: There is no significant association between achievement motivation and aggression scores of Attacker players at inter collegiate men Volleyball players

To achieve this hypothesis, the Karl Pearson's correlation coefficient technique has been applied and the results are presented in the following table.

Table: Results of correlation coefficient between achievement motivation and aggression scores of Attacker players at inter collegiate men Volleyball players

\begin{tabular}{|l|l|l|l|}
\hline \multirow{2}{*}{ variables } & \multicolumn{3}{|l|}{ Correlation coefficient between achievement motivation with } \\
\cline { 2 - 4 } & Correlation coefficient & t-value & P-value \\
\hline Aggression scores & 0.0707 & 0.3749 & 0.7106 \\
\hline
\end{tabular}

From the results of the above table, it can observed that, a non-significant and positive relationship was observed between achievement motivation and aggression scores of Attacker players at inter collegiate men Volleyball players $(\mathrm{r}=0.0707, \mathrm{p}>0.05)$ at $5 \%$ level of significance. Hence, the null hypothesis is accepted and alternative hypothesis is rejected.

Hypothesis: There is no significant association between achievement motivation and aggression scores of Setter players at inter collegiate men Volleyball players

To achieve this hypothesis, the Karl Pearson's correlation coefficient technique has been applied and the results are presented in the following table.

Table: Results of correlation coefficient between achievement motivation and aggression scores of Setter players at inter collegiate men Volleyball players

\begin{tabular}{|l|l|l|l|}
\hline \multirow{2}{*}{ variables } & \multicolumn{3}{l|}{ Correlation coefficient between achievement motivation with } \\
\cline { 2 - 4 } & Correlation coefficient & t-value & P-value \\
\hline Aggression scores & 0.2031 & 1.0975 & 0.2818 \\
\hline
\end{tabular}

From the results of the above table, it can observed that, a non-significant and positive relationship was observed between achievement motivation and aggression scores of Setter players at inter collegiate men Volleyball players $(\mathrm{r}=0.2031, \mathrm{p}>0.05)$ at $5 \%$ level of significance. Hence, the null hypothesis is accepted and alternative hypothesis is rejected.

Hypothesis: There is no significant association between achievement motivation and aggression scores of Libero players at inter collegiate men Volleyball players

To achieve this hypothesis, the Karl Pearson's correlation coefficient technique has been applied and the results are presented in the following table.

Table: Results of correlation coefficient between achievement motivation and aggression scores of Libero players at inter collegiate men Volleyball players

\begin{tabular}{|l|l|l|l|}
\hline \multirow{2}{*}{ variables } & \multicolumn{3}{l|}{ Correlation coefficient between achievement motivation with } \\
\cline { 2 - 4 } & Correlation coefficient & t-value & P-value \\
\hline Aggression scores & 0.3617 & 1.9998 & $0.0451^{*}$ \\
\hline
\end{tabular}

${ }^{*} \mathrm{p}<0.05$ From the results of the above table, it can observed that, a significant and positive relationship was observed between achievement motivation and aggression scores of Libero players at inter collegiate men Volleyball players $(\mathrm{r}=0.3617, \mathrm{p}<0.05)$ at $5 \%$ level of significance. Hence, the null hypothesis is rejected and alternative hypothesis is accepted. It means that, the achievement motivation scores are increases or decreases with increase or decrease in aggression scores of Libero players at inter collegiate men Volleyball players. 


\section{Discussion on Findings:-}

In view of the formulation of hypotheses the following results were emphasized based on the analysis of data The result of the study shows that there has no significant difference in the achievement motivation among attacker, setter, libero between these variables have better in achievement motivation

The result of the study showed that there has significant difference in the aggression among attacker, setter \& libero at inter collegiate level men volley ball players Because volley players need very quick movement for receiving and attacker the ball every second they keep on moving place to place quickness is a prime necessity in the modem volleyball both in attack and defence. That is the reason attacker, setter and libero player hove more aggressive in game.

\section{Summary:-}

The purpose of the study was to find out whether there was any significant difference in achievement motivation aggression for the Inter collegiate level attacker, setter, libero of the volley ball players. In order the achieve the purpose of the study 90 selected men volley ball players only.

To assess the level of aggression inventory questionnaire was used and the data were collected from the subjects.

To assess the level of achievement motivation the sports achievement motivation questionnaire designed and validated by Mr.kamlesh was used the data were collected from the subjects.

The collected data were put into statistical analysis. The level of significance was set at 0.05 level to find out the significance difference between means one way analysis of variance was used.

\section{Conclusion:-}

On the basis of the interpretation of the data the following appropriate conclusions are drawn from the study.

Attacker players have significant higher achievement motivation scores as compared to setter and Libero players at inter collegiate men volleyball players. $(\mathrm{F}=3.4596, \mathrm{p}<0.05)$

Attacker players have significant higher aggression scores as compared to setter and Libero players at inter collegiate men Volleyball players. $(\mathrm{F}=9.6898, \mathrm{p}<0.05)$

\section{Suggestions:-}

1. Similar study may be conducted taking to the other psychological variables.

2. Similar study may be conducted for school, state, national and university level volley ball players

3. Similar study may be conducted among different sports and games.

4. Similar study may be conducted among female volley ball players.

5. Similar study can be conducted among different age groups in different disciplines.

\section{Bibliography:-}

\section{Books:-}

$>$ Arkes H.R. and Garske J.P., Psychological Theories of Motivation ( $2^{\text {nd }}$ End,) (Monterey C.A.Brooks, 1982) p.56

$>$ Ambeim,Daniel D. "modem principle of athletic training" ,Published by The C. V Mospy company, U,S,A,( edition-1985) P.196

$>$ Burarke AG. Hayal P.S and Rajendar singh, "Relationship on Achievement motivation and Attribution to performance outcome in competitive Athletic", souvenir VII national conference of sport psychology, Thirvanandapuram.

$>$ Charles A. Bucher and Deborah A. wuest, Foundations of physical education and sports (saint Louis: Times mirror/mosby college publishers,1987),P.57

$>$ Diane L. Gill. Psychological Dynamics of sort ( IIIinois: Human kinematics publishers, Inc,. 1986),P.57

$>$ Sesai D.B., Research on Achievement motivation (London: Macmillan Book company, 1970) P.64 


\section{Journals and Periodicals:-}

$>$ Anita Ghosh, "Achievement motivation among national and international Sportsmen", Souvenir, First International and sixth national conference on sports Psychology,(New Delhi: February 1914)P.26.

$>$ Lenny B,. "women aggression in Achievement situations". Psychological bulletin, (1997)1-13.

$>$ OM kumari " motivational profile of women hockey players In India" souvenir, First International and Sixth national conference of sports psychology, ( February 1991),P,3.

$>$ Sidhy, S.R. Gangopadhyay and A.K.Uppal, "A Study of sports motivation of Indian and zimbabwen women hockey team" NIS scientific journal (1998),P.17-20. 